\title{
Influence of boundary conditions on the sound radiation and active control design of a sandwich plate with corrugated core
}

\author{
Enrico Bono ${ }^{1}$, Hui Zheng ${ }^{2}$
}

\begin{abstract}
The aim of my current research is to improve the acoustic performance of a sandwich plate with corrugated core. It finds application in high speed trains and aircraft, where a major concern for the comfort of passengers is the low frequency noise, especially after the ineffectiveness methods of geometric optimization and passive control. Active control by means of PZT patch is the approach my research will follow. When the dynamics of the structure is studied, the boundary conditions (BC) play an important role: they influence natural frequencies and mode shapes, and the way the active control should be implemented. In this paper I show my results on the vibroacoustics performance of the sandwich structure, together with the influence of $\mathrm{BC}$ on the actuator placement.
\end{abstract}

Keywords - vibroacoustics analysis, sandwich beam, uncertain BCs, PZT, active structural acoustics control

\section{Introduction}

Sandwich constructions provide several key benefits over conventional structures, such as very high bending stiffness, low weight, cost effectiveness and durability. This makes them ideal for train and aircraft or any application that involves high speeds. One of the drawbacks of the use of lightweight material is the poor acoustics properties: vibration levels could increase due to fewer mechanical connections (with respect to a uniform thick plate), leading to a reduction in damping effect.

The sound transmission loss of composite materials, and in particular sandwich panels, has been the subject of recent studies, and in particular the geometry of both faces and core has been acoustically optimized [1]. Results agree that the optimization affect the high frequency band more than other bands. The relatively small mechanical links of the core contribute dynamically to the vibration of the structure only for very high frequencies, governed by the so-called local modes. Conversely, when the global modes are taken in to account, the core parameters have much less impact on the overall vibration (and sound power). In a real application the $\mathrm{BC}$ are not always known a priori, they are a variable. Even in a controlled environment - like an experimental setup - a perfect constraint of all the degrees of freedom is impossible. [2] showed how mode shape and acoustic characteristics (such as mean square velocity, radiation efficiency and sound transmission loss) are affected by different combinations of BCs. It was concluded in particular that the influence was more evident in the acoustic properties with respect to the vibration level given by the mean square velocity.

\footnotetext{
${ }^{1,2}$ Enrico Bono, Hui Zheng

Shanghai Jiao Tong University

China
}

In this paper I will analyze in detail the effect of the stiffness of the boundaries on the dynamical behavior as well as for the radiated sound power, by means of a FE approach.

In the second part, active control design is introduced, since methods other than geometrical optimization are needed to reduce the noise levels. Passive control is the simplest method, but its efficacy is confined in a narrow frequency band [3]. It is not a practical choice for the low frequency range either, because a much thicker and heavier layer is required, losing all the effort done to design a stiff, compact and lightweight composite structure. An active control approach is a valid alternative. In particular, the use of PZT patches has been studied from many researchers, first for vibration control and also for active structural acoustic control of beams and panels [4]. It provides ease of implementation, creates a lightweight and compact system, and overcomes the disadvantages of a point force actuator (like control spillover). The actuator model that I used is derived from the classical approach, provided by Fuller and Elliott [5]. One of the most important choices when a PZT control is implemented is the position of the actuator on the structure. The decision on where exactly locate the actuator, and which length to choose, is the first important choice in an active control design. In this paper I present a method similar to the one proposed by Barboni et al. [6]. In their work, they derived an explicit dynamic influence function, and an optimal actuator length-position solution was found for different modes of vibration. Finally, I show that the boundary conditions affect this solution, demonstrating how they can directly influence an active control approach and that they cannot be neglected in a comprehensive vibroacoustics study.

\section{Dynamics and sound power}

\section{A. Finite element method}

The aluminum sandwich plate (see Figure 1) can be treated as a combination of single plates. However, following the traditional way of modeling the vibration and sound transmission of sandwich structures, the structure is assumed infinite along $\mathrm{z}$ axis. The three dimensional problem is simplified to a plane strain problem. In this way all the analysis can be performed considering the structure as a beam sandwich, and thus utilizing beam type finite elements. The dynamics in the $\mathrm{z}$ direction is neglected: this is acceptable in the present work since the stiffness in that direction is much higher - due to the core geometry - and it does not influence the low frequency range. Another simplification is given by considering the core and the plate as a unique part in the finite element formulation in the areas where they are in contact (welded). A numerical model for the corrugated core sandwich plate is created using MATLAB. I made this choice in order to have enough 


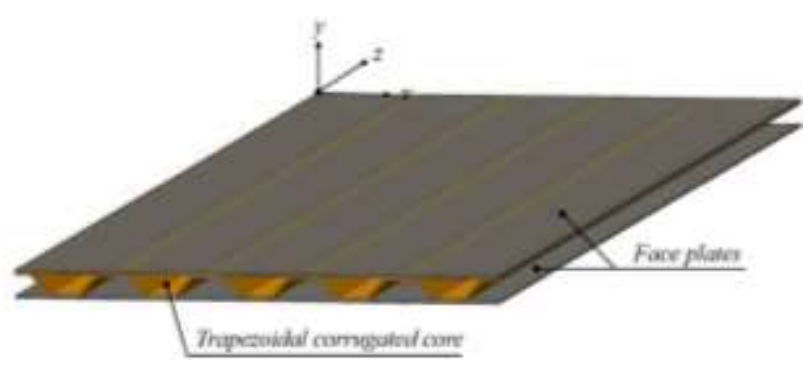

Figure 1. Sandwich plate, only the $\mathrm{x}-\mathrm{y}$ section will be considered.

freedom in a parametric study, once uncertain boundary conditions are introduced. The main disadvantage is found in drawing and meshing the structure, which is more difficult with respect to other CAD based software and limits the mesh density that I used. The structure is modeled with 92 nodes connected to form 100 beam finite elements. I built an equivalent model, but with a much refined mesh, in Abaqus, in order to verify the quality of the MATLAB code, with good matching of results.

\section{B. Natural frequencies and modes}

Since the sound transmission is strictly related to the vibration, the calculation of natural frequencies and modes shapes will be of primary importance. The boundary conditions in this first analysis are clamped on all the edges, on both sides and on both the plates, since they are the easiest to be reproduced in an experimental environment. The resulting natural frequencies are shown in Table 1, while the first modes of vibration are shown in Figure 2. In the low frequency range, the modes do not differ from the ones of a single layer slender beam: they are the so called global modes. The high range is instead dominated by the local modes. These modes depend on the peculiar geometry of the core of the sandwich panel, and therefore they differ in shape by the slender beam modes. These modes characterize a much higher frequency range with respect to the one I am interested in for the active control design.

\section{Sound radiation}

I now calculate the sound radiation caused by the vibration of the sandwich beam. The forcing term is a concentrated force of harmonic time behavior, placed in a point such that all the vibrational modes present in the range $0-1 \mathrm{kHz}$ have been excited in a uniform way. This is for an unbiased comparison between the structural vibration and the radiated sound power. In order to calculate the sound radiation, I follow the Rayleigh formulation. The reference structure and control surface filled with air are shown in the Figure 3 . The pressure distribution can therefore be obtained by the Rayleigh integral, which sums the contribution of the elemental radiator elements in which the upper beam has been decomposed

$$
p(r, \theta, t)=\frac{j \omega \rho_{0}}{2 \pi} e^{j \omega t} \int_{S_{\text {beom }}} \frac{v_{n}\left(r_{s}\right) e^{-j K R}}{R} d S_{\text {brom }}
$$

where $\omega$ is the frequency of oscillation of the elemental radiator, $\rho_{0}$ is the air density, $y_{n}$ the normal velocity of the elemental radiator, $r_{s}$ is its position according to the reference system, $r$ the radius of the control surface (constant), $R$ the distance of the elemental radiator to the
Table 1. First 8 numerical natural frequencies.

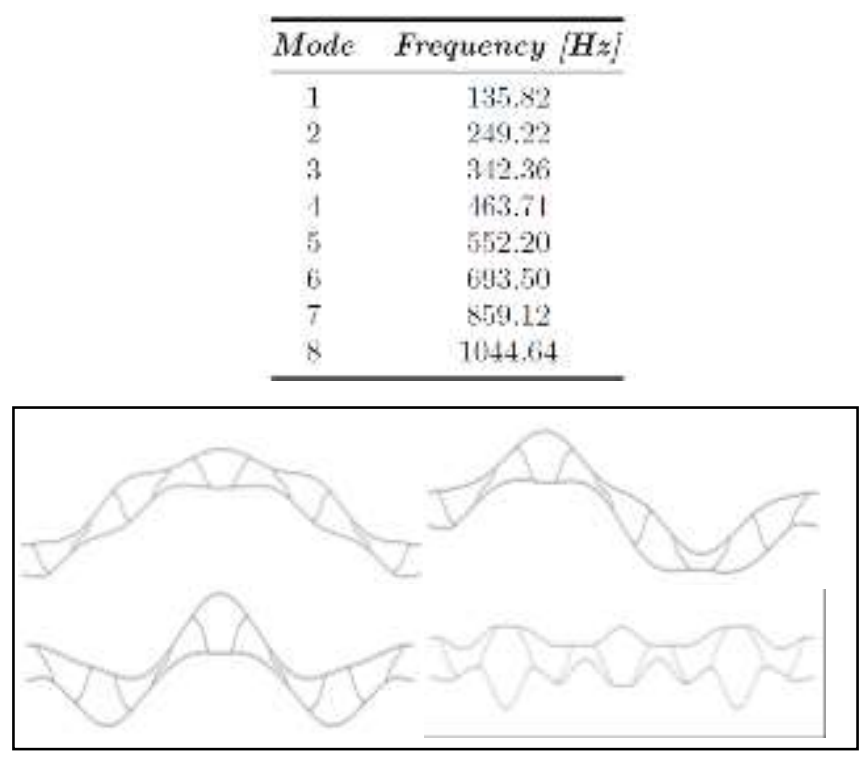

Figure 2. From top left: first three modes of vibration. In the bottom right corner an example of local mode for $1887 \mathrm{~Hz}$.

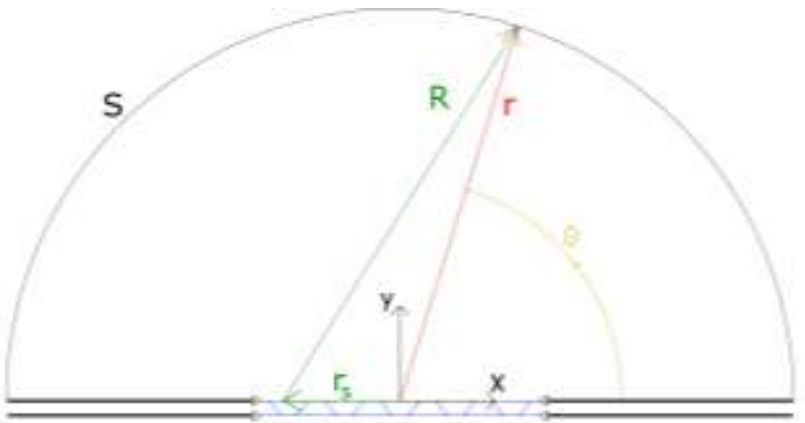

Figure 3. Control surface and reference system for the sound analysis.

point on the control surface, $\theta$ the angle between the horizontal line and $r$ and $K$ the wave number (function of the frequency and the sound velocity in air). The elemental radiators coincide with the single finite elements, and in this way I can easily obtain the displacements, as they can be calculated for the nodes. The time dependence is replaced by frequency using a Fourier transform. Furthermore, since I am considering a two dimensional case and infinite dimension along $\mathrm{z}$ axis the width of the beam is ignored, and the surface integral actually becomes a line integral. For the sound intensity I used a far field approach, such that sound and pressure are related by $p=\rho_{0} c v$, (being $\rho_{0}$ the density of air and $c$ the speed of sound). The sound power is finally obtained integrating the sound intensity over the control surface, and can be expressed conveniently in cylindrical coordinates

$$
P(\omega)=\int_{0}^{\pi} I(\theta, \omega) r d \theta=\int_{0}^{\pi} \frac{1}{2} \frac{|p(\theta, \omega)|^{2}}{\rho_{0} c} r d \theta
$$

The results of the sound power (Figure 4) show that the odd modes are the main radiators. The sound power of the even modes is many order of magnitude lower. The reason is that for low frequencies, the volume velocity is proportional to the overall sound power: the most efficient radiation mode (the velocity distribution that radiate sound 


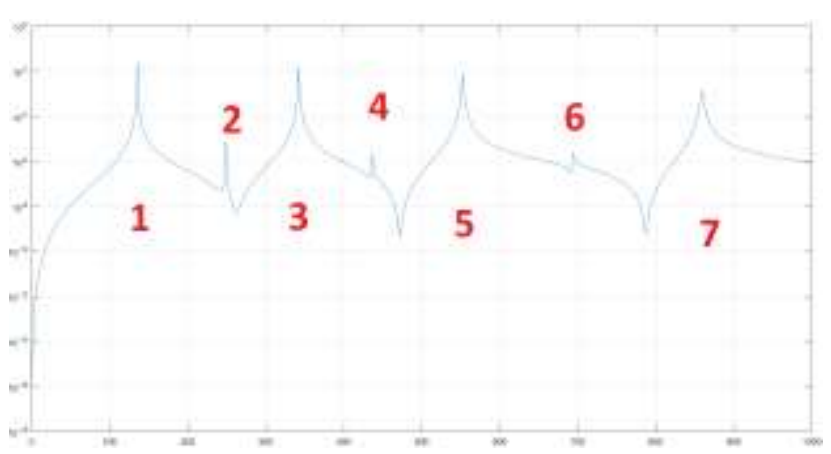

Figure 4. FRF of the radiated sound power [W] integrated over the control surface. Modes are indicated in red over the peaks.

independently from the others) in this frequency region is the first, the mono-pole type that simply express the net volume velocity. In the even mode of a beam - and, for low frequencies, also for this structure - the volume velocity of the even modes approaches zero, since there are the same number of positives and negative contributions to the velocity distribution. Since the first and third modes of vibration dominate the acoustic behavior of the structure in the range of interest, the active control will be designed in order to attenuate them, ignoring the others.

\section{Uncertain boundary conditions}

It is important to analyze how the stiffness of the boundary conditions can affect the behavior of the structure in terms of natural frequencies, mode shapes and sound radiation. I replace the ground-fixed constrains at the boundaries with displacement and torsional springs fixed to the ground. The stiffness is a parameter, and when it tends to infinite, it theoretically replicates a constrain that completely blocks a degree of freedom. The clamped-clamped condition is reproduced when it applies to every spring. In Figure 5, it is possible to observe the general configuration.

\section{A. Influence on dynamics}

In the finite element method, the natural frequencies and mode shapes are derived from an eigenvalue problem, solved for the mass and stiffness matrices of the structure. In the MATLAB built code, I iterated this process for different values of the spring stiffness, that is characterized by a precise position in the stiffness matrix, according to the degree of freedom that it governs. The results show that when stiffness of the constraints is reduced, all the natural frequencies decrease. This is in accordance with the fact that the natural frequency is roughly proportional to the square root of the structural stiffness. It has also been noticed that there is not a significant difference in varying either the top or the bottom springs. In all the considered cases for a stiffness tending to infinite, the clamped-clamped natural frequencies are approached, as for the mode shapes. The mode shapes vary together with the natural frequencies: this influence cannot be neglected in the design of a vibration control system. The low frequency range is characterized by transversal displacement of the nodes, while the longitudinal displacements can be ignored: the influence of the horizontal constrain is therefore not shown; it did not bring to clear modifications on the mode shapes. The results for the vertical constraints are shown in Figure 6 for the left and both sides respectively, and for the first and third modes. The mode shapes vary significantly, especially in the first natural frequency, and they will therefore interact with the surrounding air with different efficiencies. This

Figure 5. Schematic representation of the uncertain BCs on the edges.
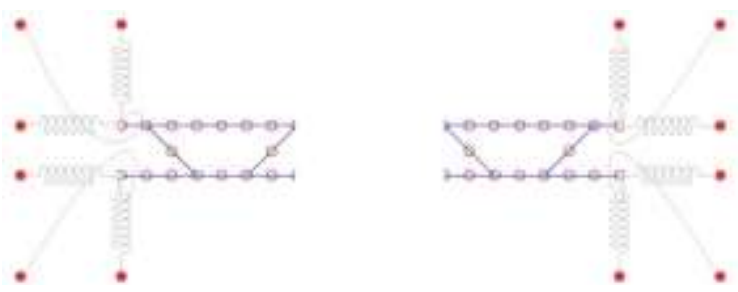

phenomenon is pointed out in the next section. As shown in Figure 7, the rotational stiffness influences only the beam shape close to the edges, without altering the central part of the structure.

\section{B. Influence on radiated sound power}

In this section, I analyze the influence of the boundary stiffness on the peaks of the sound power frequency distribution, which are the structural resonances. In particular, I show the results for the most significant case of the low frequency band, which is the first resonance (results for the third are not shown, I will give only the final considerations). The forcing term is a concentrated force with frequency equal to the first or third natural modes, which depend on the boundary stiffness as analysed in the previous section. From the results (Figure 8), it is interesting to observe that the sound power and the overall kinetic energy of the structure are not related in both the first and third resonance case. While the vibration of the structure is higher if the boundary allows a bigger vertical displacement, the sound radiated decreases. Radiation mode can help in understanding the reason. In Figure 9, the first radiation modes of a thin beam are plotted: they are velocity distributions which radiate sound independently [7]. In particular, it is shown that when the excitation frequency is sufficiently low, the size of the panel is small compared with the acoustic wavelength ( $k l$ less than 1 ), and only one radiation mode (the uniform displacement) has a significant radiation efficiency. So the decreased efficiency is due to the fact that the velocity distribution of the first structural mode for low stiffness value is similar to the second radiation mode instead of the first, decreasing its efficiency.

Considering the torsional stiffness, the opposite behavior is observed if we consider the case of $k_{\theta}$ varying on both sides (the case only one edge is considered results in a shape similar to the one of the precedent case). Here, the sound radiation is proportional to the kinetic energy. The mode shape in the case of the torsional stiffness is only modified close to the boundaries that contribute less and less to the radiation of sound as the allowed rotation decreases. 

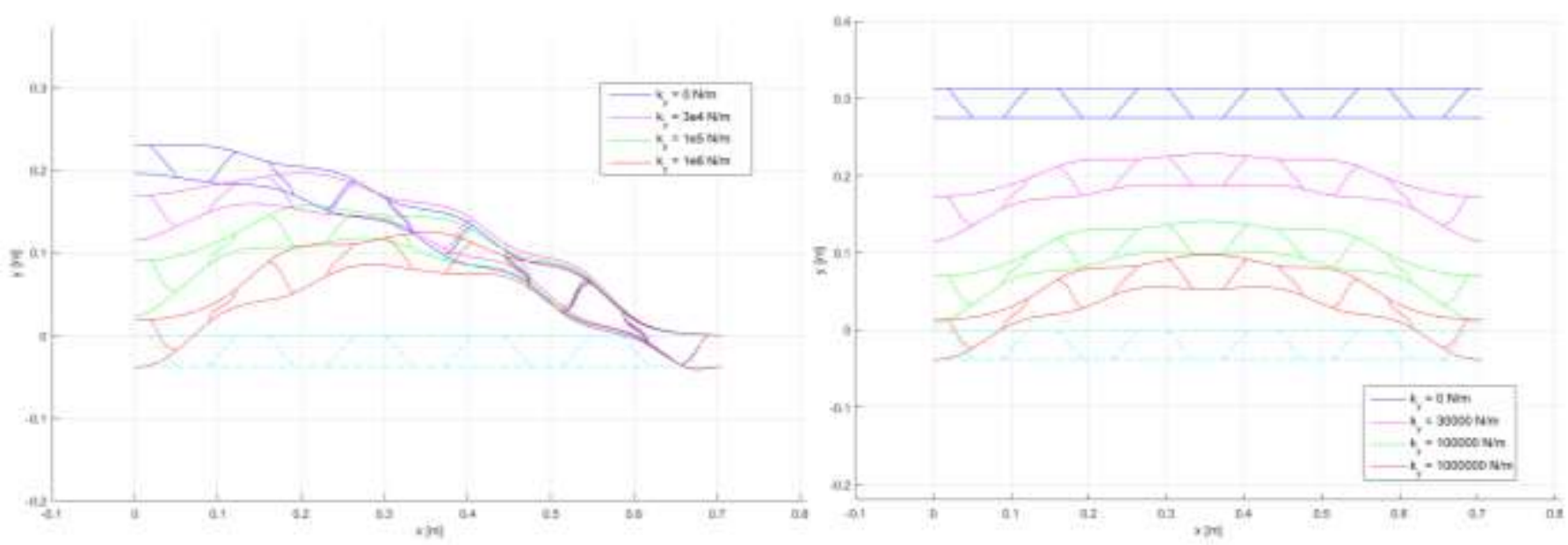

Figure 6. Different first mode shapes for different vertical stiffness of the left (on the left) and both sides (on the right).
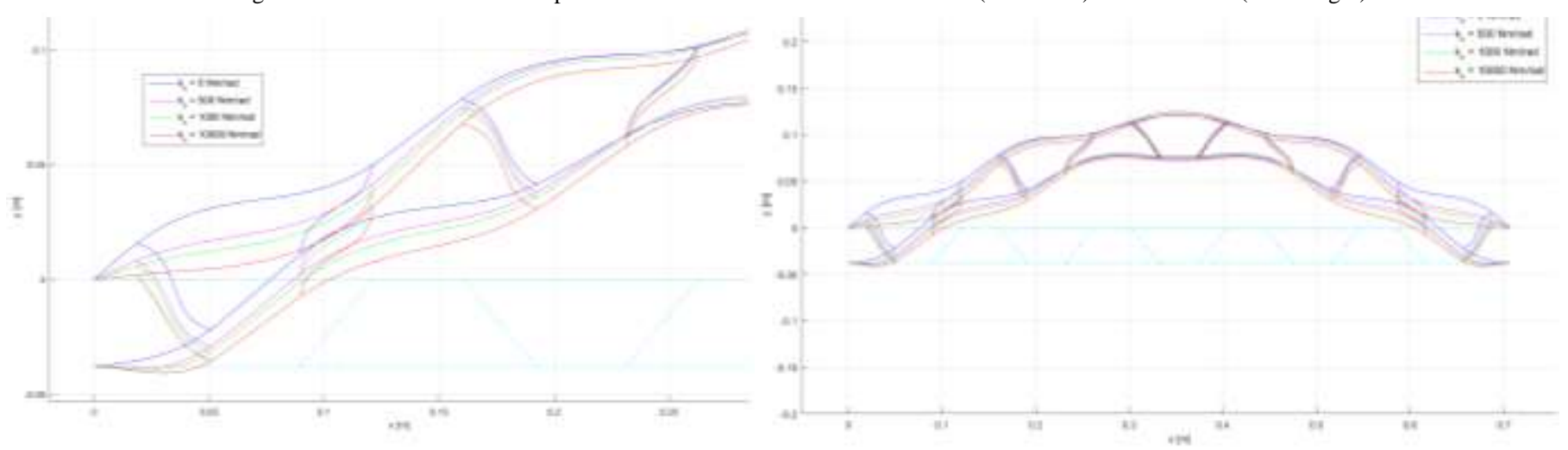

Figure 7. Different first mode shapes for different torsional stiffness. The central part is not affected from the increased stiffness.
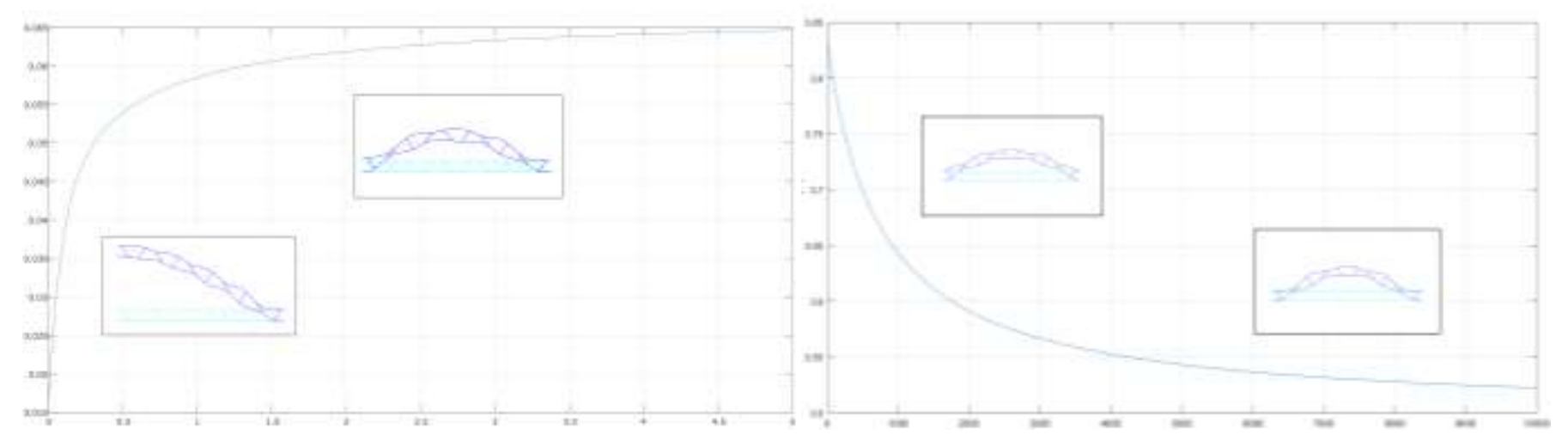

Figure 8. On the left: radiated sound power on the first resonance [W] as function of the left vertical stiffness; on the right: radiated sound power on the first resonance $[\mathrm{W}]$ as function of the torsional stiffness.
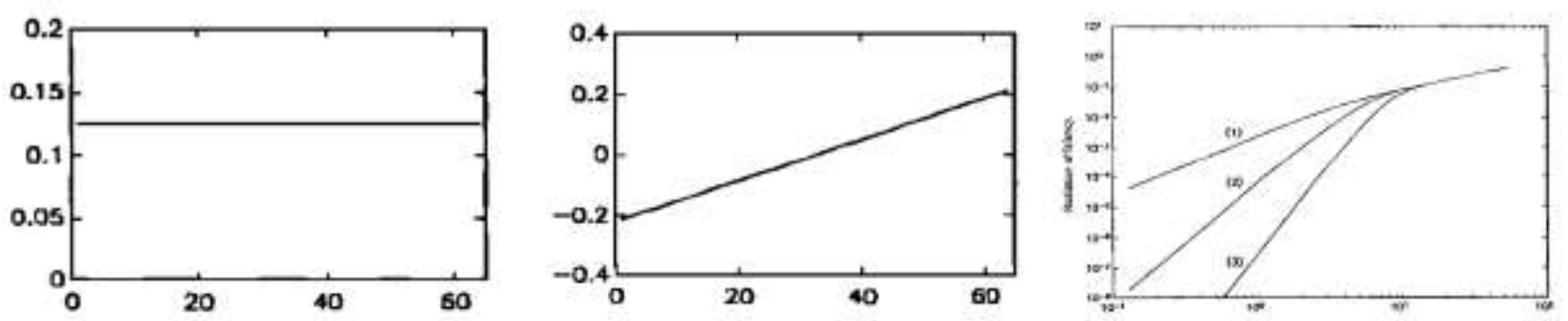

Figure 9. Starting from the left: first radiation mode; second radiation mode; efficiency of the first, second and third modes as function of $\mathrm{kl}$. 


\section{Active control}

The actuator used for active control has been chosen to be a PZT patch. The idea is to use the inverse piezoelectric effect to induce strain in the actuator by applying a certain voltage furnished from a control unit. The strain of the actuator is directly transferred to the structure as they are bonded together. The result will be a combination of forces and moments that can influence the overall dynamics and finally the sound power.

The For the actuator model I follow the theoretical formulation by Fuller and Elliott in [5]. The most important assumptions are: unidimensional problem, all of the quantities in the $\mathrm{z}$ direction are not considered as well as the Poisson effect; static approach, the PZT patch is considered without mass, no inertia influence on the structure; bounding layer between structure and actuator is considered to have infinite stiffness (perfect bonding). It is found that the induced bending of the actuator can be represented as an external load consisting of a pair of concentrated moments of opposite sign located at the actuator edges. Elliott came to this consideration starting from a simple mono-dimensional case of a rectangular beam. In the considered geometry, the section is not constant since of the corrugated core, and so the thickness depends on the position along the beam length. However, for a primary study and in order to draw some preliminary general consideration, I neglect the influence of the thickness. This allows me to follow the approach in the aforementioned reference.

\section{A. Optimal placement}

Deciding where to place the actuator in the structure is an important task: a bad placement can result in an impossibility to force the structure or it can require high gain and high voltage consumption from the control unit. In order to find the best location for the actuator, I follow a similar approach to the one proposed by Barboni et al. [6]. In this reference, the authors built a dynamic influence function that explicitly accounts for the size and the position of the actuator. The influence function is found by the modal approach, focusing on the mode we want to control. The basic concept is to find the configuration that allows the maximum work available for a certain mode, which is the maximum of the modal Lagrangian work. In particular, I concentrate on the analysis on the first mode of vibration. They [8] considered a simple beam case and therefore the analytical solution for the mode shapes was known. The complexity of a sandwich structure with corrugated core causes the analytical solution to be difficult, therefore the numerical one is considered here for the mode shapes.

From the classical FEM formulation, and using a modal approach, the forced motion of the structure is described by the uncoupled equations

$$
m_{i} \ddot{q}_{i}+c_{i} \dot{q}_{i}+k_{i}=Q_{i}
$$

For $i=1,2, \ldots, N_{D O F}$, where $m_{i}, c_{i}$ and $k_{i}$ are the modal mass, damping and stiffness of the $i^{\text {th }}$ mode. Now the actuator as forcing term has to be introduced and it is schematized in Figure 10. The PZT presence generates the concentrated moments and forces as illustrated. The virtual work is given by

\section{$\delta W=F_{0}\left(\delta u\left(x_{1}+h\right)-\delta u\left(x_{1}\right)\right)-M_{0}\left(\delta \theta\left(x_{1}+h\right)+\delta \theta\left(x_{1}\right)\right)$}

Using modal approach and substituting in the above equation and considering only the first mode, the modal amplitude is finally given by

$$
Q_{1}\left(x_{1}, h\right)=M_{0}\left(-\phi_{(x 1+h)_{1}}^{\prime}+\phi_{(x 1)_{1}}^{\prime}\right)
$$

Since the longitudinal work results zero for the first mode. Its value depends on the values of the actuator position and length thorough both the mode shape and the module of the moment/force. The explicit expression for $\mathrm{M}_{0}$ depends on the PZT physical characteristic and on the actuator placement through the thickness value. Neglecting the influence of the thickness on the absolute value of the moment and being the actuator parameters constant, the amplitude of the moment will resolve as a constant as well. It does not influence the shape of the function and can be neglected in this study. The result is finally depending only on the shape of the vibration mode, in particular to its derivative calculated in the two application points of the PZT patch. The result is plotted in Figure 11. What is important is the location of the maxima or the minima, that are equivalent for the actuator placement: a negative contribution is as valid as a positive one, it will be characterized only by an out of phase voltage. In choosing the parameters is important to consider that smaller actuators are preferred, in order to minimize the weight and ease of application on the structure. From the graph, it can also be noticed that an actuator length of $10 \mathrm{~cm}$ could be an interesting choice, since various maxima and minima are present and it is an acceptable length.

\section{B. Boundary condition influence on the actuator placement}

In the previous section, I showed how the stiffness of the boundaries influences the mode shapes. Since the modal cost function directly depends on the derivative of the first mode, the same will be true for its maximum/minimum values, i.e. the optimal placement-length pair. In particular, I want to show that the decision of the actuator position $x_{I}$ (with a fixed actuator length of $10 \mathrm{~cm}$ ) varies when the stiffness $k_{y}$ and $k_{\theta}$ are less than infinite. The iterative calculation is similar to the one used before; in this analysis, I find in each cycle the mode shape associated to the first mode, then the influence function, and finally the maximum of its absolute value. This is repeated in a stiffness range from zero very high values. For values that approach infinite, the optimal solution is the same as observed in the clamped case, which is an actuator located at $56.92 \mathrm{~cm}$ from

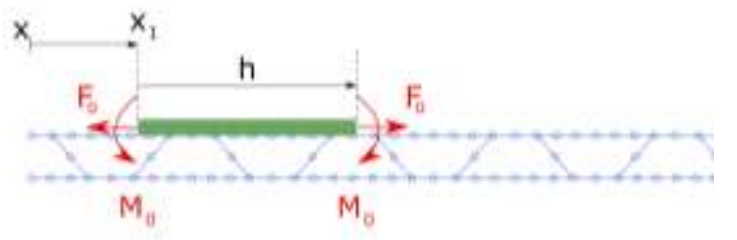

Figure 10. PZT actuator bonded to the structure (in green). 


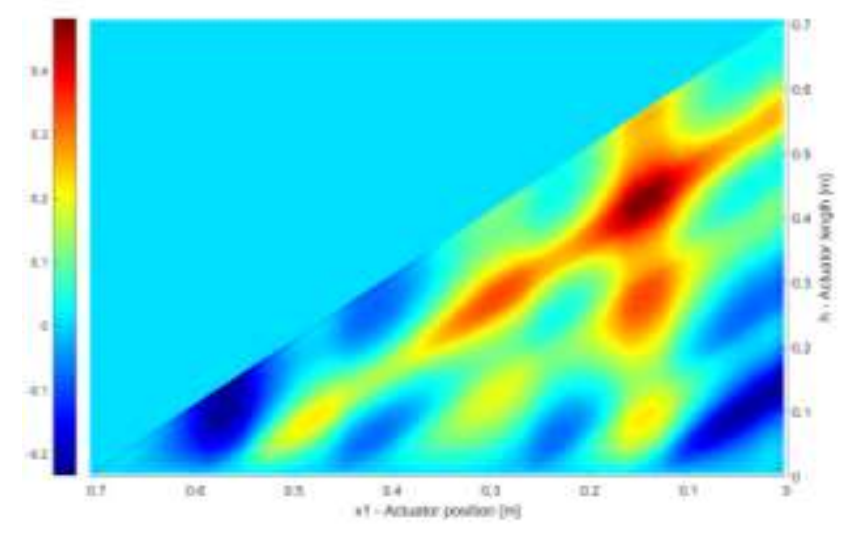

Figure 11. Dynamic influence function that represents the controllability on the first mode of vibration. It can be observed that the maximum value is found for an actuator length of $45 \mathrm{~cm}$ and $x_{1}=15 \mathrm{~cm}$ circa. A more reasonable value that with good controllability is found for $\mathrm{h}=10 \mathrm{~cm}$

the left corner of the beam. I first consider the influence of the vertical springs; these are the conclusions: when only the left side stiffness is decreased, the optimal solution differs from the ideal case in the range 5.1e $5<k_{y}<22.3 e 5 \mathrm{~N} / \mathrm{m}$. The optimal position of the actuator becomes $13.69 \mathrm{~cm}$ from the left side; when the stiffness of both sides is varied, another optimal solution of $x_{1}=46.94 \mathrm{~cm}$ is found if the stiffness is $k_{y}<7.35 \mathrm{e} 6 \mathrm{~N} / \mathrm{m}$. For the rotational spring, the results show that: if only one side is considered, the optimal solution does not change. This means that the change in the mode shape on only one side does not affect the optimal actuator placement on the other (that has a constant shape); when the rotational springs are changed on both sides, a similar solution of the vertical case is found: the same position $\left(x_{1}=46.94 \mathrm{~cm}\right)$, but for another stiffness vale, $k_{\theta}<$ $6347 \mathrm{Nm} / \mathrm{rad}$.

\section{v. Conclusions}

A numerical model for the dynamics and the radiated sound power of a sandwich plate with corrugated core has been presented. Particular attention has been paid for the boundary conditions modeling and it has been shown how they modify both the vibration and the acoustic characteristics of the structure. In the first part, I showed how the natural frequencies vary considerably: this confirmed the fact that a passive approach is not possible when the boundaries are not clearly defined, since it can cover only a narrow frequency range around the predicted resonance. It has also been shown how vibration and sound properties are not directly related. A better numerical analysis for acoustic properties predictions is possible considering a fully coupled structural-acoustic model. It is known that the fluid loading modifies the structural parameter of the plate into a model with increased mass and damping. Furthermore, an acoustic coupling between the core cavities would be able to describe the air borne sound transmission path from one side of the sandwich plate to the other [8], while only the structural borne path is modeled by the present paper.

In the second part of the paper, I presented a method based on the modal approach that finds the best actuator position-length for the controllability of the first mode of vibration. The method involves the calculation of an influence function and could also be generalized for the other modes if necessary. I have also shown how the maximum of the function is directly affected by the boundary conditions stiffness. In order to better model the PZT patch, a continuous model that accounts for the different thickness of the upper beam should be implemented in the next steps.

The next steps of my research will involve the implementation of a sensor system - composed by an array of accelerometers to detect the volume velocity - and a control unit to minimize a sensed error. Experimental validation will be done in the laboratory of Politecnico di Milano, in order to verify the accuracy of the numerical conclusion presented in this paper.

\section{Acknowledgment}

This research is supported by the National Science Foundation of China (Grant No. 51275289).

\section{References}

[1] Denli, H., and J. Q. Sun. "Structural-acoustic optimization of sandwich structures with cellular cores for minimum sound radiation." Journal of Sound and Vibration 301.1 (2007): 93-105.

[2] Zheng, Hui, and Zhiping Wei. "Vibroacoustic analysis of stiffened plates with nonuniform boundary conditions." International Journal of Applied Mechanics 5.04 (2013): 1350046.

[3] De Bedout, Juan Manuel. Adaptive-passive noise control with selftuning Helmholtz resonators. Diss. Purdue University, 1996.

[4] Gardonio, P., et al. "Analysis and measurement of a matched volume velocity sensor and uniform force actuator for active structural acoustic control." The Journal of the Acoustical Society of America 110.6 (2001): 3025-3031.

[5] Fuller, Christopher C., Sharon Elliott, and Philip A. Nelson. Active control of vibration. Academic Press, 1996.

[6] Barboni, Renato, et al. "Optimal placement of PZT actuators for the control of beam dynamics." Smart Materials and Structures 9.1

[7] Elliott, S1 J., and M. E. Johnson. "Radiation modes and the active control of sound power." The Journal of the Acoustical Society of America 94.4 (1993): 2194-2204.

[8] Wang, Yibo, Hui Zheng, and Zhiping Wei. "Finite element analysis of vibro-acoustic response of sandwich plates with corrugated cores subject to airborne excitation." INTER-NOISE and NOISE-CON Congress and Conference Proceedings. Vol. 246. No. 1. Institute of Noise Control Engineering, 2013.

About Author:

\begin{tabular}{|c|}
$\begin{array}{c}\text { Enrico Bono is a master student currently } \\
\text { attending a double degree program in } \\
\text { mechanical engineering, in the universities } \\
\text { of Politecnico di Milano and Shanghai Jiao } \\
\text { Tong University. The focus of his master } \\
\text { thesis research is active structural acoustic } \\
\text { control. }\end{array}$ \\
\hline
\end{tabular}

